\title{
Aprender con tecnologías para enseñar con tecnologías en República Dominicana. El programa República Digital Educación
}

\author{
Carlos Marcelo García ${ }^{1}$ (D) @ \\ Denia Ramona Burgos ${ }^{2}$ (D) (1) \\ Paulino Murillo Estepa ${ }^{1}$ (D) @ \\ Juan Francisco Jaspez Nero ${ }^{2}$ (D) (1) \\ ${ }^{1}$ Universidad de Sevilla, España; ${ }^{2}$ Ministerio de Educación de la República Dominicana
}

Resumen. La integración de las tecnologías digitales en los centros educativos y aulas en América Latina no es reciente. Diferentes países han desarrollado sus propias políticas, adaptadas a sus tradiciones, condiciones y posibilidades. En este artículo presentamos de forma resumida el programa República Digital Educación que, desde el año 2017, se viene implementando en la República Dominicana. El programa República Digital Educación es una iniciativa de la presidencia del gobierno dominicano y se plantea como objetivo la modernización y mejora de la calidad de la enseñanza y los aprendizajes de los estudiantes a través de la integración de las tecnologías digitales en los procesos de enseñanza y aprendizaje, así como en la gestión de los centros educativos. El programa contempla la entrega de un computador (laptop) para cada docente, equipo de gestión y estudiante, así como pizarras digitales. El programa República Digital Educación ha adoptado un modelo de formación centrada en la escuela. Ha creado la figura del facilitador (un docente del sistema educativo con altas competencias tecnopedagógicas) que trabaja permanentemente en los centros educativos. En este artículo describimos el proceso formativo desarrollado y los resultados preliminares del programa.

Palabras clave: Tecnologías digitales; formación docente; brecha digital; inclusión digital

Aprender com tecnologias para ensinar com tecnologias na República Dominicana. O programa República Digital Educación

Resumo. A integração das tecnologias digitais nas instituições de ensino e nas salas de aula na América Latina não é recente. Diferentes países desenvolveram suas próprias políticas, adaptando-as às suas tradições, condições e possibilidades. Neste artigo, apresentamos de forma resumida o programa República Digital Educación, que desde 2017 vem sendo implementado na República Dominicana. O programa República Digital Educación é uma iniciativa da presidência do governo dominicano que visa modernizar e melhorar a qualidade do ensino e da aprendizagem dos estudantes por meio da integração de tecnologias digitais nos processos de ensino e de aprendizagem, bem como na gestão das instituições de ensino. O programa inclui a entrega de um computador (laptop) para cada professor, equipe dirigente e aluno, bem como de lousas digitais. O programa de República Digital Educación adotou um modelo de formação voltado para a escola. Ele criou a figura do facilitador (um professor do sistema educacional com altas competências técnico-pedagógicas) que trabalha permanentemente em centros educacionais. Neste artigo, descrevemos o processo de formação desenvolvido e os resultados preliminares do programa.

Palabras-chave: tecnologias digitais; formação de professores; exclusão digital; inclusão digital.

Learning with ICT to teach with ICT in the Dominican Republic. The program "República Digital Educación"

Abstract. The integration of digital technologies in schools and classrooms in Latin America is not recent. Different countries have developed their own policies, adapted to their traditions, conditions and possibilities. In this article we present briefly the program "Republica Digital Educación" that is implemented in the Dominican Republic since the year 2017. The program "Republica Digital Educación" is an initiative of the Presidency of the Dominican Republic and raises aimed at the modernization and improvement of the quality of the teaching and learning of the students through the integration of technologies digital teaching and learning processes, as well as the 
management of schools. The project includes the delivery of a (laptop) computer for each teacher, equipment management and student, as well as digital whiteboards. The program "Republica Digital Educación" has adopted a model of teacher development centered on school. The figure of the facilitator (a teacher of the educational system with high techno-pedagogical competences) which permanently works in schools has been created. In this article we describe the formative process developed and the preliminary results of the program.

Keywords: Digital technologies; teacher education; digital divide; digital inclusion.

\section{INTRODUCCIÓN}

El Networked Readiness Index (NRI) de 2015 es un informe que destaca las características de los países en relación con la introducción y uso de las tecnologías en la sociedad. Utiliza cuatro criterios para valorar la situación de cada uno de los países: Ambiente (clima político, de regulación, y de innovación en las empresas); Disponibilidad (infraestructuras, de formación de las personas, de conectividad); Nivel de uso (individual, en los negocios, en el gobierno) e Impacto (económico y social). La República Dominicana, se sitúa en el puesto 94 de 143, y con una puntuación de 3,6 sobre 6. La situación diagnosticada por el índice pone de manifiesto una realidad ya conocida y que la investigación ha ido mostrando: los esfuerzos por introducir innovaciones en el sistema educativo, en especial el uso de las tecnologías digitales, no puede hacerse de forma simple y desconectada del resto de componentes que favorecen su implantación.

En los últimos dos años, la República Dominicana ha iniciado un programa denominado República Digital Educación, con el objetivo de acortar la gran brecha digital que existe en su población y que las estadísticas claramente muestran. El programa contempla una transformación de los centros educativos a través de una combinación de infraestructura tecnológica (dotación de computadores a equipos de gestión, docentes y estudiantes; instalación de pizarras digitales en aulas y de servidores en los centros educativos) y de formación docente para la integración de las tecnologías digitales. Este artículo reflexiona y presenta resultados en relación al componente formativo del proyecto República Digital Educación.

La literatura especializada en la incorporación de las tecnologías en las escuelas pone de manifiesto la necesidad de tener una visión compartida y un plan de política en relación con las TICs (Hew y Brush, 2007). Este plan de política tecnológica en educación puede tener diferentes niveles: nacional, regional, o escolar. Además se entiende como el proceso de desarrollar, revisar e implementar planes tecnológicos que permitan obtener los objetivos previstos. Un plan de política tecnológica en educación no tiene que ver sólo 
con hardware y conectividad, sino fundamentalmente con la integración de las tecnologías en los programas educativos, como veremos a continuación. Siguiendo a Vanderlinde, Braaka y Dexterc (2012), un plan de política tecnológica en educación debería estar basado en una visión compartida sobre la enseñanza y el aprendizaje y en cómo las tecnologías pueden integrarse en esta visión. El plan debe de contemplar la relación de las tecnologías con el contenido curricular y con el aprendizaje de los alumnos, debe ser dinámico y sujeto a cambios y actualizaciones a partir de su monitorización y evaluación y el plan debe ser elaborado de forma colaborativa, implicando a participar en el plan a los diferentes actores involucrados en el diseño y desarrollo del plan.

La implantación de cualquier innovación en un sistema educativo requiere de la adopción de un modelo de planificación del cambio. En este proceso han de participar los diferentes estamentos implicados en el proceso educativo. Picciano (2006) propone un modelo de planificación de las tecnologías en el que da importancia al nivel de distrito, donde los propios distritos son los que analizan las posibilidades y necesidades de tecnologías, como de recursos y procesos formativos, para posteriormente descender al nivel de escuela. Así, se hace necesario el establecimiento de un modelo de planificación tecnológica que dé respuesta adecuada y justifique las decisiones que han de adoptarse. Como comentan Barry y Nichole (2001), los planes de implantación de tecnologías normalmente han separado la dotación a los centros educativos de tecnología con la necesidad de formar a los docentes para la transformación de la práctica pedagógica con apoyo de las tecnologías. Este es un error que posteriormente tiene consecuencias difíciles de superar.

La implantación de las tecnologías en las escuelas requiere de la realización de obras de acondicionamiento para las infraestructuras tecnológicas en las escuelas. Y también hay que tener en cuenta el problema de seguridad y de mantenimiento. Estos aspectos han sido importantes en el programa República Digital Educación. En este programa se ha seguido a Malapile y Keengwe (2014), cuando plantean que la política de implantación de las tecnologías en un sistema educativo debería de tener en cuenta los siguientes elementos:

Equidad: Al considerar la implantación de las tecnologías, es preciso tomar decisiones en relación a las prioridades a atender. Una política de implantación puede favorecer a las escuelas con infraestructuras y condiciones de conectividad favorables, o bien atender prioritariamente a las escuelas de zonas pobres, rurales y con dificultad de conectividad. Estas últimas escuelas requieren esfuerzos económicos y humanos adicionales que han de imple- 
mentarse si la planificación de las tecnologías adopta los valores de equidad. Ello significa también atender a las necesidades educativas especiales en relación con las tecnologías.

Participación: La implantación de una innovación como son las tecnologías requiere de la participación de todos los estamentos educativos y sociales que de una u otra forma van a verse afectados por su implementación. Nos referimos no sólo a docentes, directores de escuelas, de regionales y distritos, sino de padres, comunidades, sindicatos, sociedad civil y sector privado.

Transparencia: La implantación de las tecnologías en las escuelas representa una alta inversión en recursos económicos, humanos y de gestión. Es necesario que las decisiones que se adopten en relación con las infraestructuras (tipos y modelos de dispositivos a comprar), como los procesos de formación y apoyo, posean la necesaria trazabilidad para facilitar la evaluación de su impacto en el sistema educativo.

Conectividad: No se trata sólo de dotar de conectividad a las escuelas, sino al sistema. A través de la inclusión de las tecnologías en las escuelas y aulas, se requiere disponer de una red de conexión.

Monitorización y evaluación: El proceso de implantación de tecnología debería contemplar el proceso de monitorización y evaluación de resultados del proyecto a través del establecimiento de indicadores claros.

Evaluación y diseño de software: La implantación de un programa de educación basado en tecnología requiere una evaluación del software a utilizar, potenciando en la medida de lo posible las opciones de "open source".

Determinación del hardware: ¿computadores o tabletas? Una decisión importante en relación con la integración de las tecnologías en las escuelas tiene que ver con qué tipo de dispositivo se elige para acceder a los recursos digitales. La tendencia, tal como nos muestra Michael Trucano, especialista del Banco Mundial, es a utilizar Tabletas dotadas con el SO Android.

Producción de contenidos digitales: La era digital son sus contenidos y en la utilización de tecnologías digitales en las escuelas, los contenidos digitales, multimedia, interactivos juegan un papel determinante. Si no hay contenidos con valor añadido, los docentes difícilmente van a utilizarlos en sus aulas. Por ello, un plan de implantación de tecnologías en las aulas y escuelas, requiere del establecimiento de una estrategia de reutilización, generación, difusión, distribución, selección, producción de contenidos digitales a todos los niveles del sistema educativo. 
Pero la integración de las tecnologías digitales en centros educativos cuyos docentes cuentan con un bajo o muy bajo nivel de competencia digital, como es el caso de la República Dominicana no es un proceso fácil. Nos hemos de plantear ¿cuáles son los factores que facilitan o dificultan la adopción de las tecnologías en especial en los países en vías de desarrollo? En un reciente artículo, Clayton Wright se decantaba por cinco factores condicionantes claves: Electricidad necesaria para poder alimentar los computadores; Conectividad, acceso a internet de banda ancha, con o sin cable; Formación de los profesores para el uso de tecnologías; Valoración de la docencia (salario, condiciones de trabajo, calidad de formación inicial, innovatividad); Sostenibilidad de la innovación con el mantenimiento de proyectos y de infraestructuras. Por otra parte, Bingimlas (2009) ha revisado los obstáculos o barreras que se producen en los procesos de integración de las TICs en educación. Una barrera se define como cualquier condición que crea dificultad de hacer progresar o conseguir los objetivos propuestos. En algunas revisiones se han diferenciado entre barreras internas (actitudes, creencias, prácticas y resistencias) y externas (acceso, tiempo, apoyo, recursos). Otras clasificaciones se han referido a dificultades a nivel de escuela (falta de eficacia de la formación, recursos, accesos) y de docentes (falta de tiempo, de confianza, resistencias al cambio).

Una primera dificultad que nos encontramos en la adopción de las tecnologías por parte de los docentes es la falta de confianza que estos tienen. Tradicionalmente los docentes han venido utilizando tecnologías: la pizarra, los mapas, el microscopio, las figuras geométricas de madera, etc. Eran tecnologías caracterizadas por su estabilidad (no cambiaban o cambiaban poco lo largo de los años), su transparencia (el docente sabía cómo funcionaban estas tecnologías) y por su especificidad (cada una servía para un objetivo concreto) (Koehler y Mishra, 2008). Sin embargo, las nuevas tecnologías resultan algo más complejas de comprender y utilizar. Son tecnologías versátiles, se pueden utilizar de diferentes formas y con diferentes objetivos. Así, un ordenador puede ser una herramienta para la comunicación, para el diseño y construcción, para la indagación, la expresión artística, etc. Son inestables, ya que cambian con mucha rapidez. Esta inestabilidad se pone de manifiesto en que el conocimiento requerido para aprender a utilizar tecnologías digitales nunca se para, puesto que la tecnología cambia continuamente. La inestabilidad de las tecnologías digitales requieren que los profesores están continuamente aprendiendo lo que puede suponer ambigüedad y frustración. Las TICs son opacas en su funcionamiento interno. ¿Quien sabe lo que pasa dentro del disco duro de un ordenador?

Los estudios muestran que los profesores a menudo tienen miedo al fracaso cuando utilizan tecnologías, fundamentalmente porque no se sienten preparados o capacitados para incorporar tecnologías en sus aulas. La segunda 
causa de rechazo por parte del profesorado del uso de las tecnologías, tiene que ver con la falta de formación y competencia. Está relacionada con la anterior; si los profesores no se perciben formados, no van a tener confianza en ellos mismos cuando utilizan tecnologías. Esta limitación es, en especial, importante en los países como República Dominicana, donde sólo recientemente, se han iniciado acciones de formación docente por parte del Ministerio de Educación. Pero no basta con desarrollar cualquier tipo de formación. Es llamativo que el informe de la OECD Teaching and Learning International Survey (OECD, 2013), muestre que a nivel de países desarrollados, las segunda y tercera necesidad de formación percibida por los docentes tenga que ver con las tecnologías educativas. La introducción de las tecnologías digitales en la formación docente ha reproducido los antiguos modelos dicotómicos (la tecnología enseñada como un elemento no integrado con el conocimiento pedagógico ni del contenido) (Tezci, 2011). Kay (2006) analizó la eficacia de cuatro estrategias en relación con la introducción de las tecnologías en la formación docente: colaboración, uso de tareas autenticas, enseñanza formal y aprendizaje exploratorio.

Otras estrategias que se han venido utilizando para introducir y sistematizar el uso de las tecnologías en la formación docente: el estudio de casos, la webquest, las discusiones asíncronas, la creación de actividades con historias digitales. Además, se han introducido algunos modelos instruccionales como el TPACK-Developing Instruccional Model de Koh y Divaharan (2011), el TPCK-IDDIRR (introducir, demostrar, desarrollar, implementar, reflexionar y revisar) de Lee y Kim (2014), o el MAGDAIRE (Modeled Analysis, Guided Development, Articulated Implementation, and Reflected Evaluation) de Chien, Chang, Yeh y Chang (2012). Un tercer elemento que influye en la adopción por parte del profesorado en las escuelas tiene que ver con la falta de accesibilidad. Pero la accesibilidad no se refiere sólo a la disponibilidad de computadores. Tiene que ver con el acceso a recursos digitales adaptados y adecuados para el nivel y disciplina que los docentes enseñan. Este es un desafío para la educación dominicana: el diseño de recursos educativos adaptados al curriculum recientemente adoptado. El cuarto elemento que, relacionado con los anteriores, desmotiva a las escuelas para la implantación de las tecnologías tiene que ver con la falta de apoyo técnico. Las dificultades técnicas y de formación que representan las tecnologías requiere que las escuelas dispongan de recursos humanos de apoyo constante y cercano para solventar las dificultades que los docentes encuentren.

En este artículo presentamos el componente formativo del programa República Digital Educación que, desde el año 2017, se viene implementando en la República Dominicana. El programa República Digital Educación es una iniciativa de la presidencia del gobierno dominicano y se plantea como objetivo la modernización y mejora de la calidad de la enseñanza y los 
aprendizajes de los estudiantes a través de la integración de las tecnologías digitales en los procesos de enseñanza y aprendizaje, así como en la gestión de los centros educativos.

\section{MÉTODOLOGÍA}

\subsection{El componente formativo del programa República Digital Educación}

El programa RDE se basa en el modelo ya implementado en otros países de América Latina (por ejemplo Perú y Uruguay) por el que se pretende acelerar el proceso de adquisición de competencias digitales en los docentes y estudiantes a través de la entrega de un computador (laptop) para cada docente, equipo de gestión y estudiante. El programa se centra en el alumnado de enseñanza secundaria, ya que en educación primaria se entrega a los centros educativos carritos dotados de tabletas, que pueden ser utilizadas por diferentes aulas. Asimismo, se ha dotado a las aulas de los centros educativos participantes de pizarras digitales, de equipos de robótica, de laboratorios de ciencias naturales y de un servidor para conectarse mediante intranet con el centro y con los servicios del Ministerio de Educación.

A lo largo del curso académico 2017-18, se ha desarrollado la primera fase del programa que ha incidido en un total de 150 centros educativos, 743 miembros de equipos de gestión, 3460 docentes, y en torno a 55000 estudiantes. Esta primera fase se ha desarrollado en cuatro regionales (provincias) del país. Uno de los primeros desafíos del programa consistió en crear las condiciones de infraestructura necesaria para permitir la conectividad. Esto ha sido especialmente dificultoso para algunos centros educativos con problemas de suministro continuo de electricidad.

Una de las características que hace diferente al programa RDE es su modelo de formación. Se ha asumido un modelo de formación centrado en la escuela. Se ha pretendido huir de modelos de formación masiva y tecnocéntrica, para centrarnos en un modelo cuyos principios han sido los siguientes:

Aprendizaje expandido: Toda acción de formación se desarrolla en diferentes momentos y contextos. Se contempla la necesidad de combinación de situaciones presenciales y no presenciales con el apoyo de los dispositivos tecnológicos. La combinación entre presencialidad y no presencialidad no es fija, sino que se acomoda en función del tipo de contenido a desarrollar y de las posibilidades de los actores de acceder a las situaciones de formación en función de la disponibilidad de desplazamientos o de conectividad. 
Aprendizaje enriquecido: Toda acción de formación aporta a los diferentes actores una amplia variedad de recursos tanto humanos como digitales que permitan acceder a buenas prácticas bien documentadas relacionadas con los contenidos y competencias a aprender.

Aprendizaje situado: Toda acción de formación requiere la toma en consideración del contexto de escuela o de aula en que cada uno de los actores participa. Así, las acciones de formación incluyen actividades que facilitan la puesta en práctica o transferencia de aprendizaje de los contenidos en las situaciones concretas en las que los actores se desempeñan. Esta será una condición imprescindible del diseño e implementación de toda acción formativa. Para ello, los Planes de Mejora elaborados por los centros educativos serán el componente estructural de la formación continua.

Aprendizaje activo: Como consecuencia de lo anterior, toda acción de formación requiere a los diferentes actores la movilización de sus conocimientos y competencias, a través del desarrollo de actividades formativas en las que deberán realizar una variedad de tareas basadas en la comprensión, diálogo, construcción de conocimiento, aplicación, indagación, producción o evaluación.

Aprendizaje colaborativo: Toda acción de formación incluye espacios formales o informales, presenciales o virtuales, a través de los cuales los diferentes actores deberán colaborar con otros actores próximos o lejanos, presenciales o virtuales para el desarrollo de actividades formativas específicas de cada acción de formación. Así, uno de los objetivos de toda acción de formación será la constitución de comunidades de práctica en torno a las diferentes temáticas y preocupaciones relevantes para la mejora escolar.

Aprendizaje mediado. Toda acción de formación incluye la mediación o acompañamiento presencial y virtual de los actores participantes, por parte de facilitadores con formación y buenas prácticas en el área específica del contenido de formación que en cada momento se desarrolle.

Aprendizaje flexible: La oferta formativa debe contemplar la necesidad de participación por parte de los diferentes actores en actividades de formación consideradas obligatorias, pero también con la posibilidad de que sean electivas, en función de los intereses y motivaciones de los sujetos implicados. 
Aprendizaje dirigido: Toda acción de formación tiene presente la necesidad de mejorar los aprendizajes de los estudiantes. Para ello establece las condiciones para que la formación en la que participan los actores tenga un impacto directo en la calidad de los aprendizajes de los estudiantes en las aulas y escuelas.

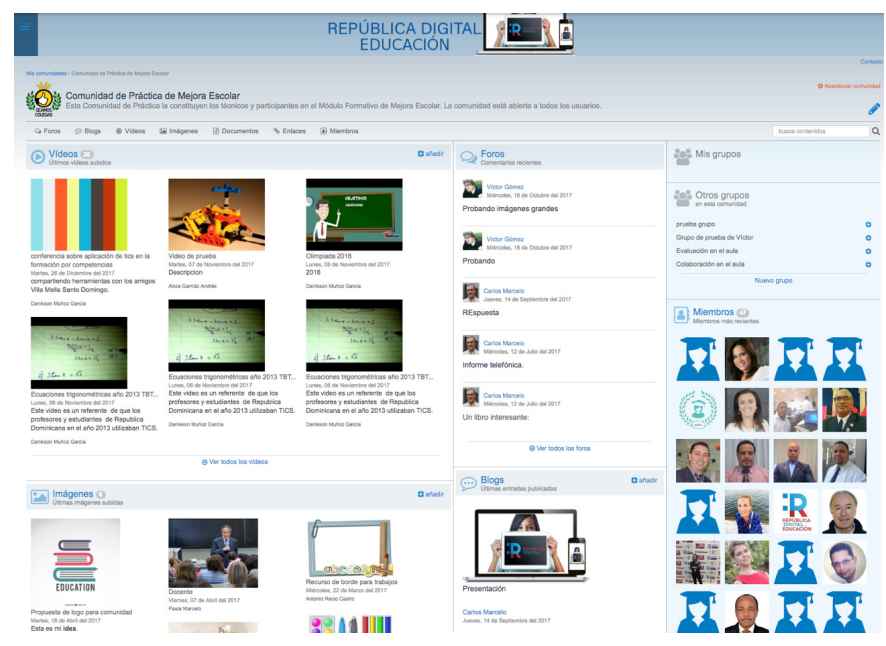

Figura 1. Plataforma desarrollada para República Digital Educación

Para hacer realidad estos principios, RDE ha diseñado una estrategia de formación centrada en la escuela para la que se necesitaban nuevos actores. El modelo de formación requiere la presencia constante de personal formativo en los centros educativos y por ello se diseñó la figura del Facilitador República Digital Educación. El facilitador es un profesional de la educación, un docente en activo del sistema educativo público no universitario, que ha desarrollado altas competencias digitales, que ha diseñado y aplicado contenidos digitales para su área de especialidad, que ha participado en proyectos de innovación docente integrando tecnologías digitales y que tiene disponibilidad para apoyar el proceso de formación de equipos de gestión y docentes participantes en el programa RDE. Los facilitadores fueron seleccionados a partir de un riguroso proceso de selección que llevó en primer lugar a un análisis de los méritos aportados por los candidatos y posteriormente la realización de un examen de demostración de competencia digital a través de una aplicación telemática (http://aptho.es). A partir de este proceso se seleccionaron 50 facilitadores, de forma que cada facilitador ha tenido asignado el acompañamiento a 3 centros educativos.

El programa de formación ha contemplado acciones de formación tanto presenciales como online para equipos de gestión y facilitadores. Para facilitar el proceso formativo se diseñó una plataforma específica que no 
sólo ofrece formación online tradicional, sino que promueve la creación de comunidades de práctica entre los docentes (http://republicadigital.edu.do). El proceso de formación se inició con la capacitación de los facilitadores. Dado que se trata de docentes con altas competencias digitales y que iban a desempeñar el papel de formadores, los seminarios formativos presenciales, así como la formación online, se centró en contenidos y metodologías vinculadas con su papel como formadores: procesos y estrategias de formación; el diagnóstico de necesidades formativas; la planificación de la formación; la evaluación de la formación; los planes de mejora con tecnologías, etc.

Las funciones asignadas a los facilitadores han sido:

- Realizan un diagnóstico de necesidades formativas en relación con las competencias digitales para cada una de sus escuelas bajo su responsabilidad.

- Establecen una relación constructiva y de confianza con el equipo directivo y el profesorado del centro.

- Diseña, en consenso con el profesorado el Plan de Formación de la escuela para la mejora de las competencias digitales y su integración en la enseñanza.

- Desarrolla acciones de formación en variadas modalidades (semanarios, talleres), dirigidas al profesorado y al equipo directivo en relación con las competencias digitales y su integración en la dirección y la enseñanza.

- Realiza observaciones de aula de docentes apoyando el uso de las tecnologías en la enseñanza.

- Realiza demostraciones en las aulas de un uso eficiente de las tecnologías digitales en el área curricular de su especialidad.

- Cumple el calendario de visitas y actividades con cada uno de los centros docentes asignados.

- Cumplimenta los impresos de seguimiento online para cada una de las actividades desarrolladas en cada escuela.

- Responde en tiempo y forma a los requerimientos de valoración e informes solicitados por la coordinación del programa.

- Informa en el tiempo establecido al Coordinador Distrital de Facilitadores en relación con las actividades desarrolladas.

- Apoya al profesorado en el diseño de actividades y evaluación de los aprendizajes de los alumnos utilizando tecnologías digitales. 
Junto con los facilitadores, se ha ofrecido formación presencial y online a los miembros de los equipos de gestión de los centros educativos participantes en el programa RDE. Así, en dos momentos, al principio y a mitad del programa, se han desarrollado seminarios presenciales centrados en facilitar la formación en competencias digitales para la dirección y gestión de centros educativos. Paralelamente a la formación presencial se han desarrollado acciones de formación online utilizando la plataforma formativa digital diseñada.

La implementación de un programa de las características de RDE ha supuesto un gran desafío en múltiples aspectos: infraestructura, equipamientos, logística, personal, formación, etc. En este artículo nos centramos en el componente pedagógico y formativo del programa RDE.

La integración de las tecnologías digitales en las aulas dominicanas no surge con el programa RDE. En Dominicana se habían implementado otros programas anteriormente, como fue el caso de COMPUMAESTRO. Sin embargo, como hemos comentado anteriormente, el modelo de formación abordado (capacitaciones masivas, puntuales y sin acompañamiento) no ha permitido una consolidación de los aprendizajes de los docentes y como consecuencia resultados esperables en la mejora de los aprendizajes de los estudiantes.

El modelo de formación del programa RDE adopta otro enfoque: para enseñar con tecnologías, los docentes han de aprender con tecnologías y este aprendizaje debe ser contextualizado, situado, acompañado y en colaboración. Y esta ha sido la principal función de los Facilitadores RDE. Los primeros meses de trabajo de los facilitadores se centraron en dar a conocer el programa RDE entre los diferentes colectivos implicados en la comunidad educativa. Estas actividades consumieron gran parte del tiempo en los inicios de la relación de los facilitadores con los docentes. Una parte importante del trabajo de los facilitadores ha estado centrada en la realización del diagnóstico de competencias digitales por parte de los docentes de los centros educativos. Otra de las actividades que han debido de realizar los facilitadores ha tenido que ver con apoyar a los miembros de los equipos de gestión para que puedan acceder a la plataforma de RDE y así poder cumplimentar los módulos formativos disponibles en línea. Esto se ha debido a que una parte de los miembros de los equipos de gestión no pudieron asistir a las sesiones de capacitación.

Una de las actividades que ha ocupado el tiempo de trabajo de los facilitadores ha sido la socialización del programa RDE ante los diferentes colectivos implicados en la comunidad educativa: docentes, estudiantes y familias. Por último, hemos de reconocer también que los facilitadores han resultado un apoyo fundamental en el proceso de entrega de los computadores en los centros educativos en los que han sido asignados. Pero la tarea de 
los facilitadores no se ha ceñido a la socialización a la gestión. Han iniciado acciones de capacitación con el profesorado de los centros educativos: Apoyo para la creación y gestión de correo electrónico por parte de los profesores; Capacitación sobre manejo del "hardware" de los equipos: mouse, teclado, pantalla...; Capacitación sobre el conocimiento del sistema operativo Windows instalado en los computadores; Capacitación en ofimática básica, especialmente en los programas Word y PowerPoint; Capacitación para la gestión de herramientas colaborativas en la suite de GoogleDrive; Acompañamiento en el aula de docentes para el apoyo con el uso de las tecnologías; Capacitación a docentes y estudiantes sobre uso saludable de Internet; Capacitación a docentes para la gestión de videos, mapas conceptuales, infografías, presentaciones y herramientas de autor.

\subsection{Modelo de evaluación del programa}

Los resultados que presentamos en este artículo corresponden a una primera evaluación de los resultados del programa República Digital Educación, desde el punto de vista de sus principales destinatarios: los docentes. Solicitamos a los docentes que se autoevaluaran en relación a la percepción de dominio de sus competencias digitales docentes. La autoevaluación de las competencias digitales por parte de los profesores ha sido una aproximación reciente en la investigación sobre integración de tecnologías digitales en la enseñanza (Knezek, G., 2017; Lázaro-Cantabrana, Usart-Rodríguez y GisbertCervera, 2019). Diferentes autores han diseñado instrumentos para establecer las percepciones de los docentes en relación a sus propias competencias digitales y el efecto de los programas en la mejora de estas competencias. En nuestra investigación elaboramos nuestro propio instrumento adecuado a las características del programa RDE y que a continuación describiremos.

\subsection{Sujetos participantes}

El Programa República Digital Educación ha implicado a un amplio número de personas: 150 centros educativos, 743 miembros de equipos de gestión, 3460 docentes, y en torno a 55000 estudiantes. Del total de sujetos implicados, en este artículo analizamos las percepciones de los docentes en relación a la adquisición de competencias digitales.

Los docentes que respondieron el cuestionario de autoevaluación de competencias digitales fueron 882 , de ellos, la mayoría eran profesoras (72,6\%). Los docentes, por lo general, tienen pocos años de antigüedad en el sistema educativo público: entre 1 y 5 años el $43.92 \%$ y entre 10 y 20 años el $26.46 \%$. Sólo el $7.94 \%$ tiene más de 20 años de experiencia. En relación 
con el nivel escolar en el que enseñan los docentes que han participado en el programa, el mayor porcentaje $(84,29 \%)$ pertenece a educación secundaria, el $23,84 \%$ a primaria y el $4.65 \%$ a educación inicial.

En relación con las características de los centros educativos participantes en el programa, el 37\% es de zona urbana; $29 \%$ de zona urbana marginal; $28 \%$ centros rurales; $4 \%$ rural aislada y $1 \%$ zona turística. Hemos de destacar el hecho de la elección de un porcentaje de en torno al $61 \%$ de los centros educativos carenciados y necesitados de un apoyo especial. Estos centros suelen tener características estructurales que hacen difícil la integración de las tecnologías debido a las dificultades de disponer de energía eléctrica constante a lo largo de todo el día, o bien por falta de conectividad.

\subsection{Instrumento}

Para conocer el grado de satisfacción de los docentes en relación con el programa República Digital Educación en el que habían participado, se diseñó un cuestionario en formato online que puede visitarse en la dirección: https://bit.ly/2lcxjza.

El cuestionario consta de 30 preguntas con diferentes formatos. En unos casos se trata de preguntas de respuesta abierta (usos que los docentes han dado a las laptops de los estudiantes durante sus clase; herramientas que los docentes han aprendido en las actividades de formación). Pero en la mayoría de los casos las preguntas eran o bien de elección (nominales) o bien valorativas (ordinales). En el cuestionario se han incluido preguntas en relación al proceso de entrega de las computadoras a los docentes y estudiantes (proceso de entrega, a quién se les ha entregado) así como sobre la instalación en las aulas de pizarras digitales. Otra parte del cuestionario pregunta a los docentes acerca de los cambios que han percibido en los estudiantes desde la entrega de las laptops (en nivel de atención, la disciplina de clase, la motivación de los estudiantes para aprender, el uso del tiempo en clase, la calidad de las tareas que deben hacer en casa, la comprensión lectora, la producción escrita, la capacidad de resolver problemas y el trabajo en equipo. En otro apartado del cuestionario se les pide a los docentes que valoren sus competencias digitales al inicio del programa y a la finalización del primer año. Además se les solicitó a los profesores que respondieran en relación a qué herramientas y recursos han aprendido a lo largo del programa formativo. Por último, se les pidió a los profesores que valoraran el trabajo desarrollado por los facilitadores del programa República Digital Educación: el trabajo del facilitador, las actividades formativas en las que han participado, la formación recibida y los aprendizajes que han obtenido. 
En el proceso de elaboración del cuestionario participaron los facilitadores del programa República Digital Educación. De esta forma, a partir de una primera propuesta, los facilitadores del programa contribuyeron a la propuesta de ítems, así como a la validación del cuestionario final. Para ello se procedió a analizar la fiabilidad de los ítems con escala ordinal del cuestionario, encontrando un alfa de Cronbach de .941 para 35 opciones de respuestas en el cuestionario.

\section{RESULTADOS}

El programa República Digital Educación pretende facilitar la integración de las tecnologías digitales en las aulas dominicanas. Para ello se implementaron un amplio número de acciones formativas centradas en la escuela, organizadas por los facilitadores y en las que los docentes podían participar. Uno de los aspectos que se ha considerado importante de conocer es en qué medida los docentes consideran que han aprendido a realizar actividades con tecnologías digitales. En la Tabla1 podemos observar cómo en una escala de 1 a 3, los docentes muestran que han aprendido ofimática básica, y también, aunque en menor medida, herramientas más elaboradas para el diseño de contenidos (creación de videos, infografías, utilización de aulas virtuales, robótica).

Tabla 1. Aprendizajes percibidos por los docentes

Aprender el funcionamiento de la computadora (utilizar el mouse, el teclado, encender, 2,71 pantalla).

Aprender el uso del sistema operativo Windows (crear, buscar, borrar carpetas y archi- 2,57 vos, comprimir archivos...)

$\begin{array}{ll}\text { Aprender a crear y utilizar el correo electrónico } & 2,58\end{array}$

$\begin{array}{ll}\text { Aprender a utilizar la pizarra digital } & 2,38\end{array}$

$\begin{array}{ll}\text { Aprender a utilizar un procesador de textos como Word } & 2,48\end{array}$

$\begin{array}{ll}\text { Aprender a hacer presentaciones con PowerPoint } & 2,33\end{array}$

$\begin{array}{lr}\text { Aprender a hacer hojas de cálculo con Excel } & 1,98\end{array}$

$\begin{array}{ll}\text { Aprender a crear videos para mis clases } & 1,99\end{array}$

Aprender a diseñar mapas mentales e infografías $\quad 1,71$

$\begin{array}{ll}\text { Aprender a utilizar aulas virtuales como Google Classroom } & 1,77\end{array}$

Aprender a utilizar herramientas de GoogleDrive como crear formularios, archivos 2 compartidos, etc.

$\begin{array}{ll}\text { Aprender a utilizar herramientas de Office } 365 & 1,67\end{array}$

Aprender Robótica $\quad 1,54$

Aprender a utilizar algún software, recurso o herramienta propia de su área curricular 2

$\begin{array}{ll}\text { Aprender sobre Internet seguro } & 2,16\end{array}$ 
Los aprendizajes percibidos anteriormente por los docentes se concretan en diferentes niveles de uso de las tecnologías digitales para la enseñanza. Nos interesó conocer si los docentes han percibido cambios en sus competencias digitales conforme el programa ha ido avanzando. Para ello identificamos cuatro niveles de competencia digital docente, que son los que aparecen en la Tabla 2. A partir de ellos se pidió a los docentes que respondieran en función de los cambios que percibían que se habían producido a partir de su participación en las acciones de formación desarrolladas en sus centros educativos. La Tabla 2 muestra el porcentaje de docentes que se identifica en cada uno de los niveles al comienzo del programa y a su finalización en julio de 2018. Como se puede observar, se producen cambios que no son drásticos en cada uno de los niveles. Quizás el más importante tiene que ver con la gran reducción de la brecha digital que se produce en los docentes que dicen situarse en el nivel de competencias bajas muy bajas. En estos docentes el nivel de progreso es evidente no sólo por las informaciones aportadas por los propios docentes sino por los facilitadores. Podemos verificar también en descenso que se produce en los docentes con niveles de competencias digitales bajo que se reduce sensiblemente. Y por otra parte, los niveles de competencia digital media y alta se ven modificados al alza.

Tabla 2. Percepción de cambios en competencias digitales por docentes

\begin{tabular}{|c|c|c|}
\hline Nivel de competencia digital de docentes & Inicio & Final \\
\hline $\begin{array}{l}\text { Muy baja. Saber cómo encender y apagar la computadora, abrir archivos, } \\
\text { navegar por internet o crear documentos. }\end{array}$ & $4,8 \%$ & $0,8 \%$ \\
\hline $\begin{array}{l}\text { Baja. Saber lo básico para crear un documento en Word, crear carpetas } \\
\text { en la computadora, crear un documento de PowerPoint básico, correo } \\
\text { electrónico. }\end{array}$ & $27,5 \%$ & $16,8 \%$ \\
\hline $\begin{array}{l}\text { Media. Crear algunos contenidos digitales como presentación animada con } \\
\text { PowerPoint, videos, imágenes; participar en una plataforma de formación } \\
\text { online; saber cómo buscar en internet; tener nociones sobre cómo utilizar } \\
\text { herramientas en la nube como formularios de GoogleDrive. }\end{array}$ & $36,5 \%$ & $41 \%$ \\
\hline $\begin{array}{l}\text { Alta. Utilizar plataformas como Edmodo o GoogleClassroom, saber cómo } \\
\text { crear y editar videos, utilizar herramientas digitales para evaluar con } \\
\text { celulares. }\end{array}$ & $11,5 \%$ & $21,3 \%$ \\
\hline Media total & 2,68 & 3,04 \\
\hline
\end{tabular}

Se ha querido conocer si las diferencias en frecuencias y porcentajes entre la valoración de la competencia digital de los docentes al inicio del programa (mes de octubre de 2017) y a la finalización de la primera fase (junio de 2018) eran estadísticamente significativas. Para ello se ha recurrido al contraste $T$ de Student para muestras relacionadas. Como se puede comprobar en la Tabla 3, el nivel de significación es de ,0, lo que significa que se ha de rechazar la hipótesis nula de que las medias correspondientes 
a las percepciones de competencia digital al inicio y fin del programa son iguales. Ello significa que hay diferencias significativas entre las percepciones de competencias digitales al final y al principio del programa.

Tabla 3. T de Student para diferencias de medias

\begin{tabular}{|c|c|c|c|c|c|c|c|}
\hline \multicolumn{5}{|c|}{ Diferencias emparejadas } & & & \multirow{3}{*}{$\begin{array}{l}\text { Sig. } \\
\text { (bila- } \\
\text { teral) }\end{array}$} \\
\hline & Desviación & Media de error & $\begin{array}{c}95 \% \text { de } \\
\text { fianza }\end{array}$ & $\begin{array}{l}\text { alo de con- } \\
\text { diferencia }\end{array}$ & & & \\
\hline Media & estándar & estándar & Inferior & Superior & $t$ & gl & \\
\hline,- 356 &, 587 & ,033 &,- 421 &,- 290 & $-10,751$ & 314 & ,000 \\
\hline
\end{tabular}

Como se ha comentado, el programa República Digital Educación tiene una orientación en los modelos "one to one" en lo que respecta a entrega de portátiles a los estudiantes. De esta forma la totalidad de los estudiantes de educación secundaria de los 150 centros educativos participantes en el programa RDE recibieron un ordenador portátil personal, que podían llevar a sus casas y mantener incluso si finalizaban su escolaridad obligatoria. De esta forma, los centros educativos, las aulas y los espacios comunes se han Ilenado de chicos y chicas con "laptops" estudiando, trabajando y, por qué no decirlo, jugando. ¿Cómo ha cambiado el ambiente del aula la presencia de un abundante número de computadores? Consultados los docentes, los resultados nos muestran las percepciones que profundos cambios en un elevado número de dimensiones que caracterizan el clima y ambiente de trabajo en las aulas de los centros participantes en el programa RDE.

En la Tabla 4 se presentan los resultados que muestran las percepciones de los docentes en relación a los cambios habidos en sus aulas a partir de la integración de ordenadores. El rango de respuesta de la escala en este ítem es de 1 el valor más bajo y 4 el mayor. Como se puede ver, los docentes muestran que es la motivación de los estudiantes, el factor que con mayor claridad se ve impactado por la presencia de los dispositivos digitales. Junto a la motivación, se perciben cambios en el trabajo en equipo, el uso del tiempo en clase y la mejora de la disciplina en el aula.

Tabla 4. Percepción de cambios en los estudiantes por el uso de tecnologías digitales

$\begin{array}{lc}\text { Items } & \text { Media } \\ \text { El nivel de atención en clase } & 3,53 \\ \text { La disciplina de clase } & 3,59 \\ \text { La motivación de los estudiantes para aprender } & 3,81 \\ \text { El uso del tiempo en clase } & 3,67 \\ \text { La calidad de las tareas que deben hacer en casa } & 3,18 \\ \text { La comprensión lectora } & 3,33 \\ \text { La producción escrita } & 3,2 \\ \text { La capacidad de resolver problemas } & 3,36 \\ \text { El trabajo en equipo } & 3,59\end{array}$




\section{DISCUSIÓN}

La integración de las tecnologías digitales en los centros educativos y aulas en América Latina no es reciente. Diferentes países han desarrollado sus propias políticas, adaptadas a sus tradiciones, condiciones y posibilidades. La enorme brecha digital que en muchos países de América Latina existía, poco a poco va reduciéndose gracias a programas institucionales como el que estamos describiendo en este artículo. Tenemos buenos ejemplos de estudios que han venido sintetizando los esfuerzos realizados en diferentes países de la región: Chile (Jara, 2013), Argentina (Vacchieri,2013), Uruguay (Vaillant, 2013), México (Barriga, 2014), CoIombia (Galvis, 2014) y Costa Rica (Muñoz et al., 2014). En el proceso de sistematización de las experiencias desarrollas, la UNESCO ha jugado un papel importante, coordinando esfuerzos para visibilizar los avances, las luces y sombras de políticas que han involucrado total o parcialmente a los sistemas educativos (UNESCO, 2013a).

No es fácil llegar a conclusiones acerca de los programas implementados. Principalmente porque la mayoría de ellos no se han mantenido en el tiempo como para poder realizar valoraciones basadas en evidencias consolidadas. Algunos de los estudios que se han desarrollado llegan a conclusiones que pueden causar controversia. Por ejemplo, Beuermann, Julian, Cueto, Malamud, y Cruz-Aguayo (2015) y Cristia, Ibarrarán, Cueto, Santiago, Severín, E. (2017) analizaron el impacto de la política "Un ordenador por niño" en Perú, encontrando que si bien el nivel de uso tanto en la escuela como en el hogar se incrementaba en los alumnos que habían recibido dispositivos, los niveles de rendimiento en matemáticas y lengua no diferían significativamente de aquellos niños a los que no se les había entregado computadores. Resultados similares aportan de Melo, Machado y Miranda (2014) en relación con el Plan Ceibal desarrollado en Uruguay.

Los resultados que presentamos en este artículo corresponden a los inicios de la implementación del programa República Digital Educación en la República Dominicana. Hemos intentado mostrar los esfuerzos realizados para huir de la demagogia o malentendido, según el cual la simple dotación de laptops a los estudiantes provocaría una mejora en los aprendizajes. Sabemos desde hace mucho tiempo que los docentes cuentan (OECD, 2005). Y sabemos que si no se presta especial atención a la capacitación de los docentes, los dispositivos electrónicos podrán tener un primer y fugaz momento de motivación y esperanza, pero son los docentes los principales protagonistas, responsables y artífices del cambio en los modelos de enseñanza que provoquen mejoras en los aprendizajes. 
Por ello, el modelo pedagógico implementado en el programa RDE ha puesto hincapié en la formación docente centrada en la escuela. Con unos principios a los que nos referimos a lo largo de este artículo: contextualización, horizontalidad, aprendizaje en la práctica, acompañamiento en terreno por iguales (facilitadores), formación presencial y online. Estos principios, implementados en una primera fase del programa, han permitido mejorar las competencias digitales de los docentes, como hemos presentado.

El programa RDE tiene unos enormes desafíos para su institucionalización definitiva. La gran inversión económica que requiere un programa "uno a uno" va asociada a la necesaria mejora de la gestión de los procesos tanto en lo que se refiere a la dotación de los equipos como a su mantenimiento. Y ello en un país en el que hay escuelas con dificultades para disponer de energía eléctrica 24 horas y como consecuencia con problemas de conectividad. Estos desafíos están poniendo a prueba la capacidad del sistema educativo, de sus agentes y de su profesorado. La valoración favorable del programa tanto de las familias, los equipos de gestión, los docentes (incluido su sindicato, la ADP), los estudiantes y la sociedad civil, están sirviendo para impulsar este movimiento que persigue acortar la brecha digital que tradicionalmente ha caracterizado a la República Dominicana.

No puede haber mejora de los resultados de aprendizaje de los estudiantes sin que los docentes mejoren su cualificación y competencia. En este sentido, la revolución que está teniendo lugar en muchos centros educativos de República Dominicana, de la mano del programa República Digital Educación, está sentando las bases de una mayor exigencia y compromiso para el profesorado en lo que respecta a la mejora de aprendizaje de los alumnos. Esperamos que con la ampliación del programa RDE a nuevas escuelas y regionales los efectos positivos que hemos mostrado en este artículo se vean ampliados y reconocidos.

\section{Referencias bibliográficas}

Beuermann, D., Julian, C., Cueto, S., Malamud, O. y Cruz-Aguayo, Y. (2015). One Laptop Per Child at Home: Short-Term Impacts from a Randomized Experiment in Peru. American Economic Journal: Applied Economics, 7(2), 53-80.

Bingimlas, K. (2009). Barriers to the Successfull Integration of ICT in Teaching Learning Environments: A review of the Literature. Eurasia Journal of Mathematics, Science and Technology Education, 5(3), 235-245.

Chien, Y. T., Chang, C. Y., Yeh, T. K. y Chang, K. E. (2012). Engaging pre-service science teachers to act as active designers of technology integration: A MAGDAIRE framework. Teaching and Teacher Education, 28(4), 578-588. 
Cristia, J., Ibarrarán, P., Cueto, S., Santiago, A. y Severín, E. (2017). Technology and Child Development: Evidence from the One Laptop Per Child program. American Economic Journal: Applied Economics, 9(3), 295-320.

De Melo, Gioia, Machado, Alina and Miranda, Alfonso (2014). The Impact of a One Laptop Per Child Program on Learning: Evidence from Uruguay. Discussion paper series. IZA DP N. 8489. Forschungsinstitut zur Zukunft der Arbeit Institute for the Study of Labor. http://ftp.iza.org/dp8489.pdf.

Díaz Barriga, F. (2014). Las políticas TIC en los sistemas educativos de América Latina: caso México. Buenos Aires: UNICEF.

Galvis, A. (2014). Las políticas TIC en los sistemas educativos de América Latina: caso Colombia. Buenos Aires: UNICEF.

Hew, K. F. y Brush, T. (2007). Integrating technology into K-12 teaching and learning: current knowledge gaps and recommendations for future research. Educational Technology Research and Development, 55(3), 223-252.

Jara, I. (2013). Las políticas TIC en los sistemas educativos de América Latina: caso Chile. Buenos Aires: UNICEF.

Kay, R. (2006). Evaluating Strategies Used To Incorporate Technology Into Preservice Education: A review of the Literature. Journal of Research on Technology in Education, 38(4), $385-410$

Koehler, M. y Mishra, P. (2008). Introducing TPCK. In Handbook of Technological Pedagogical Content Knowledge (TPCK) for Educators, (pp. 3-29). New York: Routledge.

Koh, J. H. L. y Divaharan, S. (2011). Developing pre-service teachers' technology integration expertise through the TPACK-developing instructional model. Journal of Educational Computing Research, 44(1), 35-58.

Lee, C. J. y Kim, C. (2014). An implementation study of a TPACK-based instructional design model in a technology integration course. Educational Technology Research and Development, 62(4), 437-460.

Malapile, S. y Keengwe, J. (2014). Information Communication Technology planning in developing countries. Education and Information Technologies, 19(4), 691-701.

MINERD. (2013). Marco de Formación Continua. Una perspectiva articuladora para una escuela de calidad. Santo Domingo: Ministerio de Educación. Instituto Nacional de Formación y Capacitación del Magisterio.

Muñoz, L., Brenes, M., Bujanda, M. E., Mora, M., Núñez, O. y Magaly Zúñiga. (2014). Las políticas TIC en los sistemas educativos de América Latina: caso Costa Rica. Buenos Aires: UNICEF.

OECD. (2005). Teachers matter: attracting, developing and retaining effective teachers. Paris: OECD.

OECD. (2013). Teaching and Learning International Survey. Paris: OECD.

Tezci, E. (2011). Factors that influence pre-service teachers' ICT usage in education. European Journal of Teacher Education, 34(4), 483-499.

Tondeur, Jo; van Braak, Johan; Ertmer, Peggy A.; Ottenbreit-Leftwich, Anne (2017). Understanding the Relationship between Teachers' Pedagogical Beliefs and Technology Use in 
Education: A Systematic Review of Qualitative Evidence. Educational Technology Research and Development, 65 (3),555-575.

UNESCO. (2013a). Uso de TIC en educación en América Latina y Caribe. Análisis regional de la integración de las TIC en la educación y de la aptitud digital. París: UNESCO.

UNESCO (2013b). Enfoques estratégicos sobre las TICs en educación en América Latina y EI Caribe. Santiago: Oreal/Unesco.

Vacchieri, A. (2013). Las políticas TIC en los sistemas educativos de América Latina: caso Argentina. Buenos Aires: UNICEF.

Vaillant, D. (2013). Las políticas TIC en los sistemas educativos de América Latina. Caso Uruguay. Buenos Aires: UNICEF.

Vanderlinde, R., Braaka, J. van y Dexterc, S. (2012). ICT policy planning in a context of curriculum reform: Disentanglement of ICT policy domains and artifacts. Computers \& Education, 58(4), 1339-1350.

Wright, C. (2014). 5 Key Barriers to Educational Technology Adoption in the Developing World. Educational Technology Debate, April. https://bit.ly/1hHCqNK 